\title{
Dexamethasone promotes IL-4-induced alternative activation at PPARY point, instead of upstream STAT6 in BV2 microglial cells
}

\section{Zongfeng Chen}

Army Medical University https://orcid.org/0000-0002-9643-6321

\section{Liang Zhang}

Department of Spinal Surgery, Army Medical Center, Army Medical University of PLA

\section{Xin Xue}

Department of Spinal Surgery, Army Medical Center, Army Medical University of PLA

Peng Liu

Department of Spinal Surgery, Army Medical Center, Army Medical University

\section{Xiang Yin}

Department of Spinal Surgery, Army Medical Center, Army Medical University of PLA

\section{Yu Xiang}

Department of Spinal surgery, Army Medical Center, Army Medical University of PLA

Mingyong Liu ( $\nabla$ mingyong_liu@163.com )

Department of Spinal Surgery, Army Medical Center, Army Medical University of PLA

Jianhua Zhao ( $\square$ zhaojianhua1964@yahoo.com )

Department of Spinal Surgery, Army Medical Center, Army Medical University of OLA

\section{Research}

Keywords: Dexamethasone, Microglia, Alternative activation, STAT6, PPARY

Posted Date: June 18th, 2020

DOl: https://doi.org/10.21203/rs.3.rs-33766/v1

License: @ (1) This work is licensed under a Creative Commons Attribution 4.0 International License. Read Full License 


\section{Abstract \\ Background}

Microglia are innate immune effector cells in the central nervous system and play an extremely important role in the physiological processes of the central nervous system. When microglia are activated, there are two polarization states, M1 and M2 phenotype. Dexamethasone is a glucocorticoid widely used in clinical practice, which pharmacological effects are mainly anti-inflammatory, anti-toxic. However, whether Dexamethasone affects polarization state of microglia is unknown. In this study, we investigate the effect of Dexamethasone on IL-4-induced alternative activation in murine BV-2 microglial cells.

\section{Methods}

BV-2 cells were incubated with Dexamethasone alone, IL-4 alone, or the combination of Dexamethasone and IL-4. Western blot and immunofluorescence were performed to detect protein levels of alternative activation markers arginase 1 (Arg1), found in inflammatory zone 1 (FIZZ1). Moreover, we investigated the effects of Dexamethasone on IL-4 induced activation of signal transducer and activators of transcription 6 (STAT6) and peroxisome proliferator-activated receptor-gamma (PPARY).

\section{Results}

Dexamethasone promoted IL-4 induced microglia alternative activation by increasing the expression of Arg1 and FIZZ1. Dexamethasone also enhanced the expression of PPARY. These effects were reversed by RU486 (a Dexamethasone antagonist). Further, the effects of Dexamethasone and IL-4 on Arg1 and FIZZ1 were blocked by the application of GW9662 (a PPARY antagonist).

\section{Conclusions}

Our studies confirm that Dexamethasone promotes IL-4 induced alternative activation via STAT6/PPARY signaling pathways in microglia. At the same time, it was confirmed that Dexamethasone acts on PPARY instead of STAT6. These findings support that Dexamethasone has a therapeutic potential for neuroinflammatory diseases via alternative activation.

\section{Background}

Microglia are considered to be the resident macrophages, which play a critical role in inflammatory reactions of the central nervous system (CNS)[1]. They are activated multiple states: M1 phenotype and M2 phenotype, during diverse conditions, such as trauma, inflammation, and infection. Generally, M1 microglia, also called classical phenotype, participate a pro-inflammatory role through secretion of proinflammatory factors, including TNF-a, IL-1, IL-6[2-6]. M2 microglia, also called alternative phenotype, 
play an anti-inflammatory role, and promote tissue repair through phagocytosis of protein aggregates and cell debris[2-7]. Therefore, suppression of the M1 phenotype and promotion of the M2 phenotype has been considered as a potential strategy in trauma and inflammatory therapy in CNS.

Identifying the microglia phenotype is critical for studying the function of microglia. specific M1 versus M2 markers have been examined in the context of determining the microglia phenotype. Arg1 is a typical marker for M2 microglia activation that participates in arginine metabolism[8]. FIZZ1 (also known as RELMa) belongs to the resistin-like molecule (RELM) family of secreted mammalian proteins[9]. Both Arg1 and FIZZ1 could be induced in IL-4 mediated M2 classical activation pathway.

Corticosteroids (GC) are widely used clinically for anti-inflammatory and immunosuppressive agents. Dexamethasone (Dex), a synthetic glucocorticoid, has been demonstrated to suppress the LPS-induced ROS production and NO release, then suppress the LPS-induced M1 phenotype activation in BV2 microglia[10,11]. Microglia showed a reduction of phagocytosis function, pro- and anti-inflammatory cytokine production, and cell proliferation after microglia exposed persistently to glucocorticoid (GC)[12]. However, the effect of Dexamethasone on microglia M2 polarization and molecular mechanisms is still unknown.

Here, we investigated the impact of Dexamethasone on IL-4-induced expression of M2 markers in BV-2 microglial cells and the signaling pathways involving in these processes. Our data show that Dexamethasone up-regulates expression of Arg1 and FIZZ1 in IL-4-treated microglial cells depending on STAT6 and PPARY signaling pathways.

\section{Methods}

\section{Cell culture}

The murine microglia cell line BV-2 was maintained at $25 \mathrm{~cm}^{2}$ culture bottles in DMEM ( Gibco, Grand Island, NY, USA) supplemented with 10\% FBS (Gibco, Grand Island, NY, USA), $100 \mathrm{U} / \mathrm{ml}$ penicillin and 100 $\mu \mathrm{g} / \mathrm{ml}$ streptomycin (Beyotime, Shanghai, China) at $37^{\circ} \mathrm{C}$ in a humidified atmosphere of $95 \%$ air and $5 \%$ $\mathrm{CO}_{2}$. BV2 cells were plated on 12-well culture plates at about a density of $1.0 \times 10^{6}$ per well for protein extraction, at $0.8 \times 10^{6}$ per well on a 12-well plate for immunofluorescence. After 12 hours of plating, BV2 cells were treated with different treatments.

BV-2 cells were incubated with $1 \mu \mathrm{M}$ of Dexamethasone (Cayman Chemical, Ann Arbor, MI, USA) for 60 minutes before the addition of $10 \mathrm{ng} / \mathrm{ml}$ murine recombinant IL-4 protein (Sino Biological Inc., Beijing, China).

To investigate the change in expression of Arg1, FIZZ1, STAT6, or PPARY, the cells were treated with 10 $\mu M$ RU486 (GenScript Corporation100) or $1 \mu$ M GW9662 (SigmaAldrich, St. Louis, MO, USA) before the treatment with Dexamethasone for 60 minutes. 


\section{Protein extraction}

After handled, the cells were washed three times with PBS, and were directly lysed with SDS-PAGE Sample Loading Buffer $(2 x)$ on ice and denatured at $100^{\circ} \mathrm{C}$ for 10 minutes. The samples were stored at $-20^{\circ} \mathrm{C}$ for later use.

\section{Western blot analysis}

Protein samples were electrophoresed on sodium dodecyl sulfate-polyacrylamide gels and then transferred onto a polyvinylidene difluoride membrane (Millipore, Schwalbach, Germany). The transformed membrane was blocked with QuickBlock ${ }^{\text {TM }}$ Blocking Buffer for Western Blot (Beyotime, Shanghai, China) for one hour and incubated with primary antibodies overnight at $4{ }^{\circ} \mathrm{C}$. The primary antibodies used were as follows: anti-Arg1 (1:1000, Abcam, Shanghai, China), Fizz1 (1:1000, Abcam, Shanghai, China), anti-PPARy (1:1000, Cell Signaling Technology, Massachusetts, America), antiphospho-STAT6 (1:1000, Cell Signaling Technology, Massachusetts, America), anti- $\beta$-Actin (1:1,000, Zhongshan Goldbridge Biotechnology, Beijing, China). The membrane was washed three times with PBST for ten minutes and incubated with Peroxidase-conjugated Goat anti-mouse IgG (1:2500, Zhongshan Goldbridge Biotechnology, Beijing, China), Peroxidase-conjugated Goat anti-mouse IgG (1:2500, Zhongshan Goldbridge Biotechnology, Beijing, China), Peroxidase-Conjugated Rabbit anti-Goat IgG (1:2500, Zhongshan Goldbridge Biotechnology, Beijing, China) at room temperature for two hours. ChemiDoc $^{\text {TM }}$ XRS+ System with Image Lab ${ }^{\text {TM }}$ Software(Bio-Rad, California, America) was used to detect immunoreactive bands. The intensity of protein bands after Western blot was quantitated by using Quantity One Version 4.6.3 Image software (Bio-Rad, Hercules, CA, USA) and normalized against proper loading controls.

\section{Immunocytochemistry}

BV-2 cells were cultured on sterile glass coverslips and treated according to the experimental design. Wash PBS three times, every 5 minutes. Afterward, cells were fixed with $4 \%$ paraformaldehyde in PBS. Then, cells were blocked with QuickBlock ${ }^{\text {TM }}$ Blocking Buffer for Immunol Staining (Beyotime, Shanghai, China) for 30 minutes and incubated with primary antibodies overnight at $4^{\circ} \mathrm{C}$. Wash PBS three times, every 5 minutes. After washing, cells were incubated with Cy3-labeled Donkey Anti-Goat IgG (1:500, Beyotime, Shanghai, China) or Alexa Fluor 555-labeled Donkey Anti-Rabbit IgG (1:500, Beyotime, Shanghai, China) for one hour and counterstained with 4, 6-diamidino-2-phenylindole (DAPI, Beyotime, Shanghai, China) for the identification of nuclei. After washing with PBS, the coverslips were mounted with antifade mounting medium (Beyotime, Shanghai, China) on slides, and the cells were observed with an Olympus immunofluorescence microscope (Olympus, Tokyo, Japan).

The primary antibodies used were as follows: anti-arginase-1 (1:50, Abcam, Shanghai, China), Fizz1 (1:50, Abcam, Shanghai, China), anti-PPARy (1:50, Cell Signaling Technology, Massachusetts, America), antiphospho-STAT6 (1:50, Cell Signaling Technology, Massachusetts, America). 


\section{Statistical analysis}

Data are expressed as means \pm SEM of the indicated number of independent experiments. Statistical significance between multiple groups was analyzed by one-way ANOVA. Statistical analysis was performed using the SPSS software version 13.0 (SPSS Inc., Chicago, IL, USA). P $<0.05$ was considered statistically significant.

\section{Results}

\section{Dexamethasone promotes IL-4-induced alternative activation of BV-2 microglial cells}

Firstly, we evaluated the effects of Dexamethasone on IL-4-induced microglia alternative activation; BV-2 cells were treated with $1 \mu \mathrm{M}$ Dexamethasone for 60 minutes and stimulated with IL-4 $(10 \mathrm{ng} / \mathrm{ml})$ for 36 hours. The expression of Arg1 and FIZZ1 were analyzed to evaluate the alternative activation of BV2 cells. We tested the protein levels of Arg1 and FIZZ1 using Western blot analysis. As shown in Figure 1A and B, IL-4 stimulation significantly enhanced Arg1 and FIZZ1 protein levels in BV-2 cells $(P<0.01)$. Treatment with Dexamethasone and RU486 alone did not affect Arg1 and FIZZ1 protein levels $(P>0.05)$; while a combination of Dexamethasone and IL-4 increased expression of Arg1 and FIZZ1 in BV-2 cells compared to IL-4 treatment alone. Immunofluorescence staining demonstrated that Arg1 and FIZZ1 staining intensity increased after IL-4 treatment, pretreatment with Dexamethasone further increased the staining intensity (Figure 1C). To evaluate the effects of Dexamethasone, a Dexamethasone antagonist RU486 $(2 \mu \mathrm{M})$ was administrated 60 minutes before treatment with Dexamethasone. The effects in response to Dexamethasone were reversed by RU486 (Figure 1A-B).

\section{Dexamethasone promotes IL-4-induced activation of PPARY}

STAT6/PPARy plays a critical role in the IL-4 dependent induction of Arg1 and FIZZ1[13-15]. As Dexamethasone promotes IL-4 induced expression of Arg1 and FIZZ1 (Figure 1), we investigated the involvement of STAT6/PPARY activation in these effects.

As shown in Figure 2A, IL-4 increased PPARy protein levels. Dexamethasone alone failed to activate PPARY $(P>0.05)$. However, the combination of Dexamethasone and IL-4 further increased PPARY protein levels compared to treatment with IL-4 alone, the effects of Dexamethasone were abolished by pretreatment with RU486.

We also examined the effects of Dexamethasone on IL-4 stimulated phosphorylated-STAT6 in BV-2 cells. Treatment with Dexamethasone and RU486 alone did not influence the IL-4-induced expression of STAT6 $(P>0.05$, Figure $2 B)$.

Immunofluorescence staining demonstrated that PPARy staining intensity increased after IL-4 treatment, pretreatment with Dexamethasone further increased the staining intensity (Figure 2C ). 
we used GW9662 (a PPARY antagonist) to identify the role of PPARY activation induced by IL-4 and Dexamethasone[16]. $2 \mu \mathrm{M}$ GW9662 was administrated 30 minutes before treatment with Dexamethasone, PPARy expression was analyzed. GW9662 decreased PPARy protein levels in IL-4 and Dexamethasone treated BV-2 cells (Figure 3A).

Immunofluorescence staining demonstrated that PPARy staining intensity decreased after GW9662 treatment (Figure 3B ).

\section{PPARY is essential for induction of Arg1 and FIZZ1 by IL-4 and Dexamethasone}

Dexamethasone promotes IL-4-induced PPARy activation, these facts suggest, but do not prove, that PPARY is required for the effects of Dexamethasone and IL-4 on Arg1 and FIZZ1 induction. To directly test the effects of PPARy, BV-2 cells were pretreated with or $2 \mu \mathrm{M}$ GW9662 for 30 minutes to inhibit the PPARy signaling pathway and stimulated with Dexamethasone and IL-4 for 36 hours.

Immunofluorescence and Western blot were performed to determine Arg1 and FIZZ1 expression. As expected, Arg1 and FIZZ1 induction by Dexamethasone, Arg and FIZZ1 were markedly blunted by inhibition of PPARY (Figure 4).

\section{Discussion}

In this study, we first found that Dexamethasone enhances the IL-4 induced M2 polarization in BV-2 microglial cells. The expression of Arg1 and FIZZ1, IL-4-induced expression of two major alternative activation markers, increase when pretreated with Dexamethasone before IL-4 treated. Furthermore, Dexamethasone enhanced the effects of IL-4 on PPARy signaling pathways, administering GW9662 before Dexamethasone and IL-4 blunted the induction of Arg1 and FIZZ1. These results verify that Dexamethasone can promote IL-4-induced M2 polarization in BV2 cells via STAT6/PPARy signaling pathways. Also, Dexamethasone plays a role at PPARY point, instead of upstream STAT6.

After spinal cord injury, early treatment with steroids can promote the recovery of nerve function and reduce the scope of permanent paralysis[17-19]. High-dose methylprednisolone steroid therapy is the only pharmacologic therapy shown to have efficacy in phase three randomized trials when administered within eight hours of injury[20]. However, an aqueous solution of methylprednisolone is unstable[21], and in this study, we chose a more stable and 7 times more powerful glucocorticoid analog, Dexamethasone.

In the central nervous system, microglia are usually maintained in a resting state. When activated, they have been defined as two specific activation states: M1 (classical activation) and M2 (alternative activation) in vitro[22]. In this study, we chose Arg1 and FIZZ1 as markers for microglia M2 state. Arg1 expression in macrophages is silent until signals from Th2 cytokines such as IL-4 and IL-13[23]. In the cytoplasm, Arginase catalyzes the hydrolysis of L-arginine to L-ornithine and urea, which comprises the final step of the urea cycle. This cycle provides the principal route for the disposal of nitrogenous waste from protein catabolism. Apart from Arg1, FIZZ1 is another marker for alternative activation[24]. Found in inflammatory zone 1 (FIZZ1), also known as resistin-like moleculea (RELMa), a member of the newly 
described cysteine-rich secreted family of FIZZ/RELM.7-9. FIZZ1 is considered to be relevant to profibrosis[25] and regulating inflammation[26]. First, we examined the effect of IL-4 and Dexamethasone on Arg1 and FIZZ1 expression. In our study, treatment with Dexamethasone alone had no effect on Arg1 and FIZZ1 expression in microglia, while pretreatment with Dexamethasone enhanced the effect of IL-4 on Arg1 and FIZZ1 expression. This indicated that Dexamethasone may be possible to promote the resolution of neuroinflammation by regulating microglia polarization. The effect of Dexamethasone was reversed by the use of Dexamethasone antagonist RU486.

The synergistic effect of Dexamethasone and IL-4 on Arg1 and FIZZ1 expression may be explained by the enhancement of IL-4 induced STAT6 / PPARy activation by Dexamethasone. IL4 induces the expression of Arg1 and FIZZ1, depending on the activation of STAT6/PPARY. After IL4 stimulated microglia, STAT6 was phosphorylated, transferred to the nucleus and bound to the Jmjd3 promoter, and activated the activation of the M2 marker gene [27]. STAT6 can also regulate the activity of the nuclear receptor PPARY [28]. PPARy plays an important role in the replacement activation of macrophages and microglia; PPARY agonists can directly stimulate the expression of Arg1 and FIZZ1 [29-32]. In this study, Dexamethasone could not enhance IL4-induced phosphorylation of STAT6 but could increase IL4-induced PPARY activity. $A B C$ et al. Reported that pioglitazone can increase the expression of Arg1 and Ym1 by activating PPARY, thereby promoting the M2 polarization of microglia [30]. In this study, the PPARY antagonist GW9662 can reduce the synergistic effect of Dexamethasone and IL-4 on Arg1 and FIZZ1 expression, proving that PPARy is necessary for Dexamethasone and IL-4-induced microglial M2 polarization. These results indicate that Dexamethasone relies on the STAT6 / PPARy signaling pathway to enhance IL-4 induced activation of microglia M2 subtypes (Fig. 5).

\section{Conclusions}

The results showed that Dexamethasone promoted IL-4 induced microglia replacement activation by activating the STAT6 / PPARy signaling pathway. The promoting effect of Dexamethasone on the replacement activation of microglia may be one of the possible mechanisms of Dexamethasone treatment of spinal cord injury.

\section{Abbreviations}

Arg1: arginase 1; BSA: bovine serum albumin; CNS: the central nervous system; DAPI: 6-diamidino-2phenylindole; DMEM: Dulbecco's modified Eagle's medium; Fizz1: found in inflammatory zone 1; IL: interleukin; LPS: lipopolysaccharide; PBS: phosphate-buffered saline; PPARY: peroxisome proliferatoractivated receptor gamma; STAT6: signal transducer and activators of transcription 6; TBE: Tris-borateEDTA; TBST: Tris-buffered saline containing 0.05\% Tween-20; DEX: Dexamethasone; TNF: tumor necrosis factor.

\section{Declarations}




\section{Ethics approval and consent to participate}

Not applicable

\section{Consent for publication}

All authors of this article have read and approved the final version of the manuscript.

\section{Availability of data and materials}

The raw data supporting the conclusions of this manuscript will be available by the authors, without undue reservation, to any qualified researcher.

\section{Competing interests}

The authors declare that they have no competing interests.

\section{Funding}

This work were Supported by the National Natural Science Foundation of China(81972161, 81972068 and 81501883).

\section{Authors' contributions}

Mingyong Liu, Jianhua Zhao and Zongfeng Chen designed the experiment; Zongfeng Chen, Liang Zhang, Xin Xue, Liu Peng, Wang Chenxu, and Xiang Yu experimented; Zongfeng Cheng processed the data and wrote the paper, and Mingyong Liu Jianhua Zhao modified the paper.

\section{Acknowledgments}

We thank Prof. Xiuwu Bian (Institute of Pathology and Southwest Cancer Center, Southwest Hospital, Army Medical University), for his excellent technical support. We also thank Prof. Xiuwu Bian for the gift of BV2 microglia cell.

\section{References}

1. Casano AM, Peri F. Microglia: multitasking specialists of the brain. Dev Cell. 2015;32:469-77.

2. Benarroch EE. Microglia: Multiple roles in surveillance, circuit shaping, and response to injury. Neurology. 2013;81:1079-88.

3. Beynon SB, Walker FR. Microglial activation in the injured and healthy brain: what are we really talking about? Practical and theoretical issues associated with the measurement of changes in microglial morphology. Neuroscience. 2012;225:162-71.

4. Varnum MM, Ikezu T. The classification of microglial activation phenotypes on neurodegeneration and regeneration in Alzheimer's disease brain. Arch Immunol Ther Exp (Warsz). 2012;60:251-66. 
5. Colton C, Wilcock DM. Assessing activation states in microglia. CNS Neurol Disord Drug Targets. 2010;9:174-91.

6. Gordon S, Martinez FO. Alternative activation of macrophages: mechanism and functions. Immunity. 2010;32:593-604.

7. Marshall SA, McClain JA, Kelso ML, Hopkins DM, Pauly JR, Nixon K. Microglial activation is not equivalent to neuroinflammation in alcohol-induced neurodegeneration: The importance of microglia phenotype. Neurobiol Dis. 2013;54:239-51.

8. Lisi L, Ciotti GM, Braun D, Kalinin S, Curro D, Dello RC, Coli A, Mangiola A, Anile C, Feinstein DL, Navarra P. Expression of iNOS, CD163 and ARG-1 taken as M1 and M2 markers of microglial polarization in human glioblastoma and the surrounding normal parenchyma. Neurosci Lett. 2017;645:106-12.

9. Tang Y, Le W. Differential Roles of M1 and M2 Microglia in Neurodegenerative Diseases. Mol Neurobiol. 2016;53:1181-94.

10. Huo Y, Rangarajan P, Ling EA, Dheen ST. Dexamethasone inhibits the Nox-dependent ROS production via suppression of MKP-1-dependent MAPK pathways in activated microglia. Bmc Neurosci. 2011;12:49.

11. Hinkerohe D, Smikalla D, Schoebel A, Haghikia A, Zoidl G, Haase CG, Schlegel U, Faustmann PM. Dexamethasone prevents LPS-induced microglial activation and astroglial impairment in an experimental bacterial meningitis co-culture model. Brain Res. 2010;1329:45-54.

12. Park MJ, Park HS, You MJ, Yoo J, Kim SH, Kwon MS. Dexamethasone Induces a Specific Form of Ramified Dysfunctional Microglia. Mol Neurobio/ 2018.

13. Pourcet B, Pineda-Torra I. Transcriptional regulation of macrophage arginase 1 expression and its role in atherosclerosis. Trends Cardiovasc Med. 2013;23:143-52.

14. Welch JS, Escoubet-Lozach L, Sykes DB, Liddiard K, Greaves DR, Glass CK. TH2 cytokines and allergic challenge induce $\mathrm{Ym} 1$ expression in macrophages by a STAT6-dependent mechanism. J Biol Chem. 2002;277:42821-9.

15. Gallardo-Soler A, Gomez-Nieto C, Campo ML, Marathe C, Tontonoz P, Castrillo A, Corraliza I. Arginase I induction by modified lipoproteins in macrophages: a peroxisome proliferator-activated receptorgamma/delta-mediated effect that links lipid metabolism and immunity. Mol Endocrinol. 2008;22:1394-402.

16. Gallardo-Soler A, Gomez-Nieto C, Campo ML, Marathe C, Tontonoz P, Castrillo A, Corraliza I. Arginase I induction by modified lipoproteins in macrophages: a peroxisome proliferator-activated receptorgamma/delta-mediated effect that links lipid metabolism and immunity. Mol Endocrinol. 2008;22:1394-402.

17. Miekisiak G, Latka D, Jarmuzek P, Zaluski R, Urbanski W, Janusz W. Steroids in Acute Spinal Cord Injury: All But Gone Within 5 Years. World Neurosurg. 2019;122:e467-71.

18. Dhaliwal P. Spinal Cord Injury, Steroids, and Latin America. World Neurosurg. 2016;90:636-7. 
19. Rouanet C, Reges D, Rocha E, Gagliardi V, Silva GS. Traumatic spinal cord injury: current concepts and treatment update. Arq Neuropsiquiatr. 2017;75:387-93.

20. Bracken MB. Steroids for acute spinal cord injury. Cochrane Database Syst Rev. 2012;1:D1046.

21. Fraser C, Mays A. Steroids. Rahway: Merck \& CO., Inc.; 1986.

22. Boche D, Perry VH, Nicoll JA. Review: activation patterns of microglia and their identification in the human brain. Neuropathol Appl Neurobiol. 2013;39:3-18.

23. Pauleau AL, Rutschman R, Lang R, Pernis A, Watowich SS, Murray PJ. Enhancer-mediated control of macrophage-specific arginase I expression. J Immunol. 2004;172:7565-73.

24. Orihuela R, McPherson CA, Harry GJ. Microglial M1/M2 polarization and metabolic states. Br J Pharmacol. 2016;173:649-65.

25. Martins V, Gonzalez DLSF, Wu Z, Capelozzi V, Phan SH, Liu T. FIZZ1-induced myofibroblast transdifferentiation from adipocytes and its potential role in dermal fibrosis and lipoatrophy. Am $\mathrm{J}$ Pathol. 2015;185:2768-76.

26. Nair MG, Du Y, Perrigoue JG, Zaph C, Taylor JJ, Goldschmidt M, Swain GP, Yancopoulos GD, Valenzuela DM, Murphy A, et al. Alternatively activated macrophage-derived RELM-\{alpha\} is a negative regulator of type 2 inflammation in the lung. J Exp Med. 2009;206:937-52.

27. Ishii M, Wen H, Corsa CA, Liu T, Coelho AL, Allen RM, Carson WT, Cavassani KA, Li X, Lukacs NW, et al. Epigenetic regulation of the alternatively activated macrophage phenotype. Blood. 2009;114:3244-54.

28. Szanto A, Balint BL, Nagy ZS, Barta E, Dezso B, Pap A, Szeles L, Poliska S, Oros M, Evans RM, et al. STAT6 transcription factor is a facilitator of the nuclear receptor PPARgamma-regulated gene expression in macrophages and dendritic cells. Immunity. 2010;33:699-712.

29. Odegaard JI, Ricardo-Gonzalez RR, Goforth MH, Morel CR, Subramanian V, Mukundan L, Red EA, Vats D, Brombacher F, Ferrante AW, Chawla A. Macrophage-specific PPARgamma controls alternative activation and improves insulin resistance. Nature. 2007;447:1116-20.

30. Mandrekar-Colucci S, Karlo JC, Landreth GE. Mechanisms underlying the rapid peroxisome proliferator-activated receptor-gamma-mediated amyloid clearance and reversal of cognitive deficits in a murine model of Alzheimer's disease. J Neurosci. 2012;32:10117-28.

31. Cuartero MI, Ballesteros I, Moraga A, Nombela F, Vivancos J, Hamilton JA, Corbi AL, Lizasoain I, Moro MA. N2 neutrophils, novel players in brain inflammation after stroke: modulation by the PPARgamma agonist rosiglitazone. Stroke. 2013;44:3498-508.

32. Bouhlel MA, Derudas B, Rigamonti E, Dievart R, Brozek J, Haulon S, Zawadzki C, Jude B, Torpier G, Marx N, et al. PPARgamma activation primes human monocytes into alternative M2 macrophages with anti-inflammatory properties. Cell Metab. 2007;6:137-43.

\section{Figures}




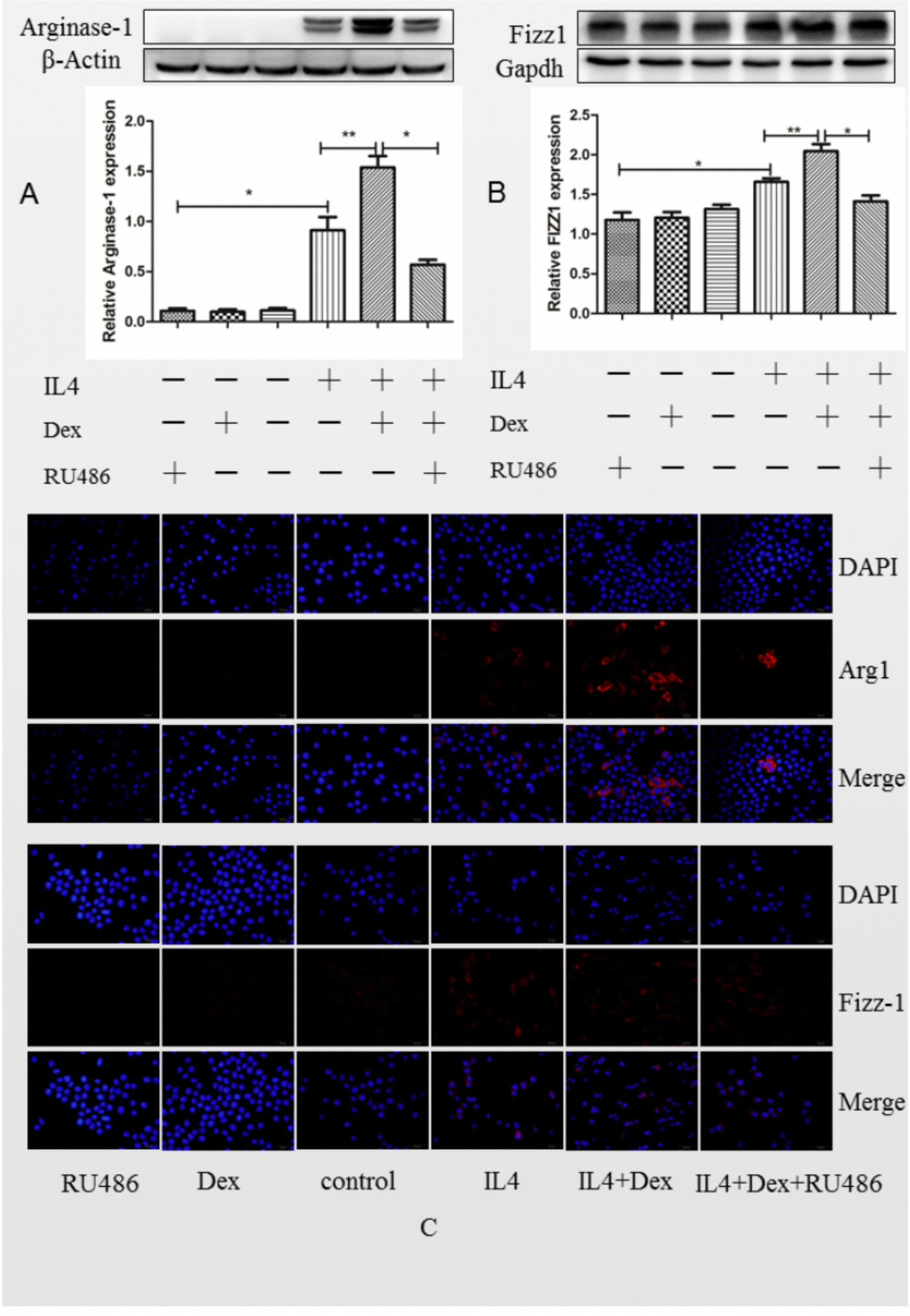

Figure 1

Dex promotes the IL-4-induced alternative activation of BV-2 cells. BV-2 cells were respectively treated with $1 \mu \mathrm{M}$ Dex for 60 minutes, $2 \mu \mathrm{M}$ RU486 and stimulated with IL-4 $(20 \mathrm{ng} / \mathrm{ml})$ for 36 hours. The expression of Arg1 and FIZZ1 were analyzed to evaluate the alternative activation of BV2 cells. We tested the protein levels of Arg1 and FIZZ1 using Western blot analysis (A, B). A representative result from three independent experiments is shown. Quantification for Arg1 and FIZZ1 was normalized by $\beta$-Actin and 
Grapdh respectively. Data are presented as mean \pm SEM for three independent experiments. Asterisks indicate statistically significant difference $\left({ }^{\star} P<0.01,{ }^{\star *} P<0.05\right)$. Representative images of BV-2 cells with positive immunofluorescence staining for Arg1 and FIZZ1 (C). Scale bars indicate $20 \mu \mathrm{m}$.

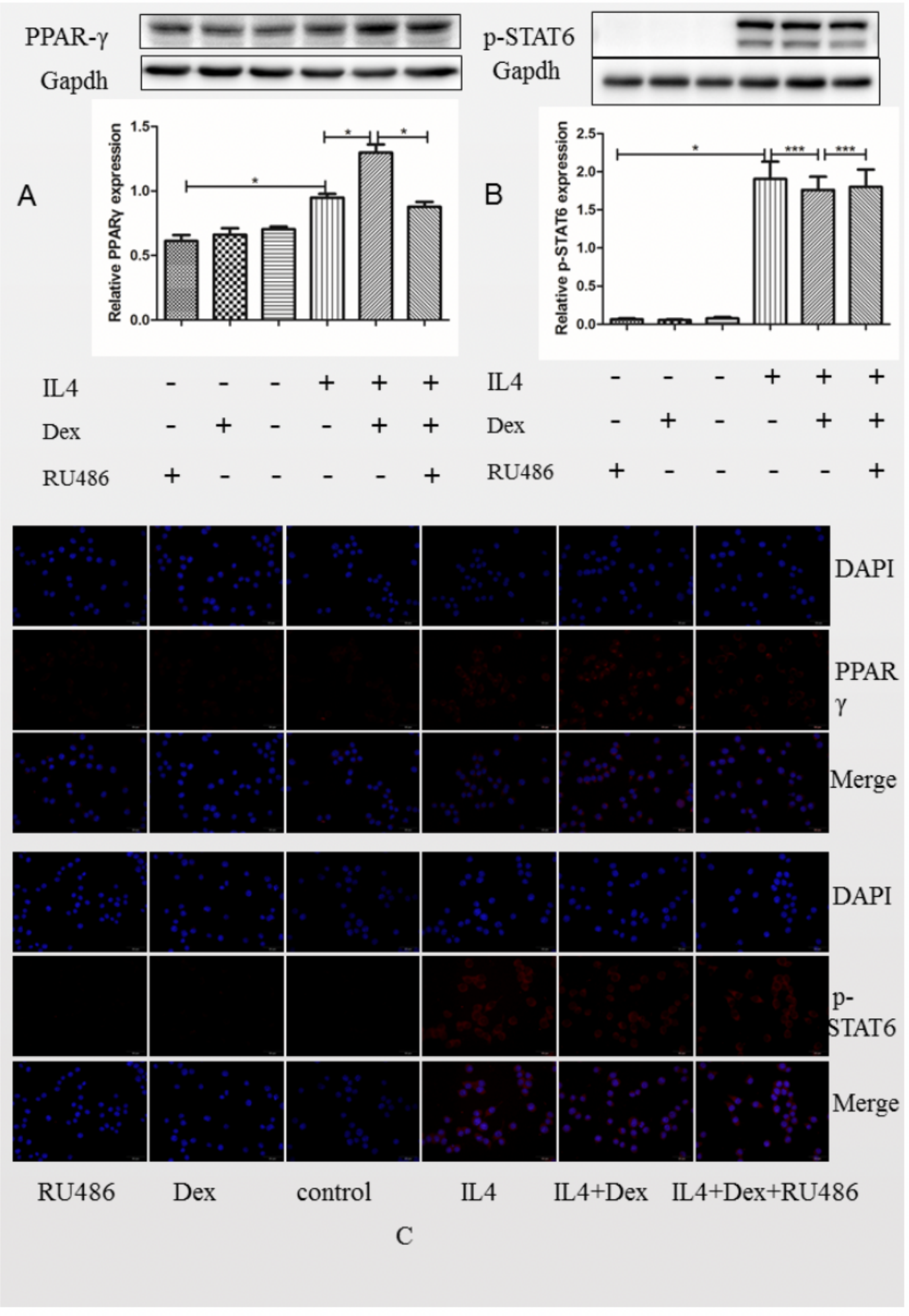

Figure 2

Dex enhances IL-4 induced PPARy activation. BV-2 cells were treated with $1 \mu \mathrm{M}$ Dex, $2 \mu \mathrm{M}$ RU486, IL-4 (20 $\mathrm{ng} / \mathrm{ml}$ ) or combination of them. Levels of phosphorylated STAT6 and PPARY were detected by Western 
blot 24 hours after IL-4 stimulation. A representative result from three independent experiments is shown. Quantification of phosphorylated STAT6 was normalized by $\beta$-actin. Data are presented as mean \pm SEM for three independent experiments. Asterisks indicate significant difference ( $\left.{ }^{*} P<0.05, \star \star \star P \unrhd 0.05\right)$.

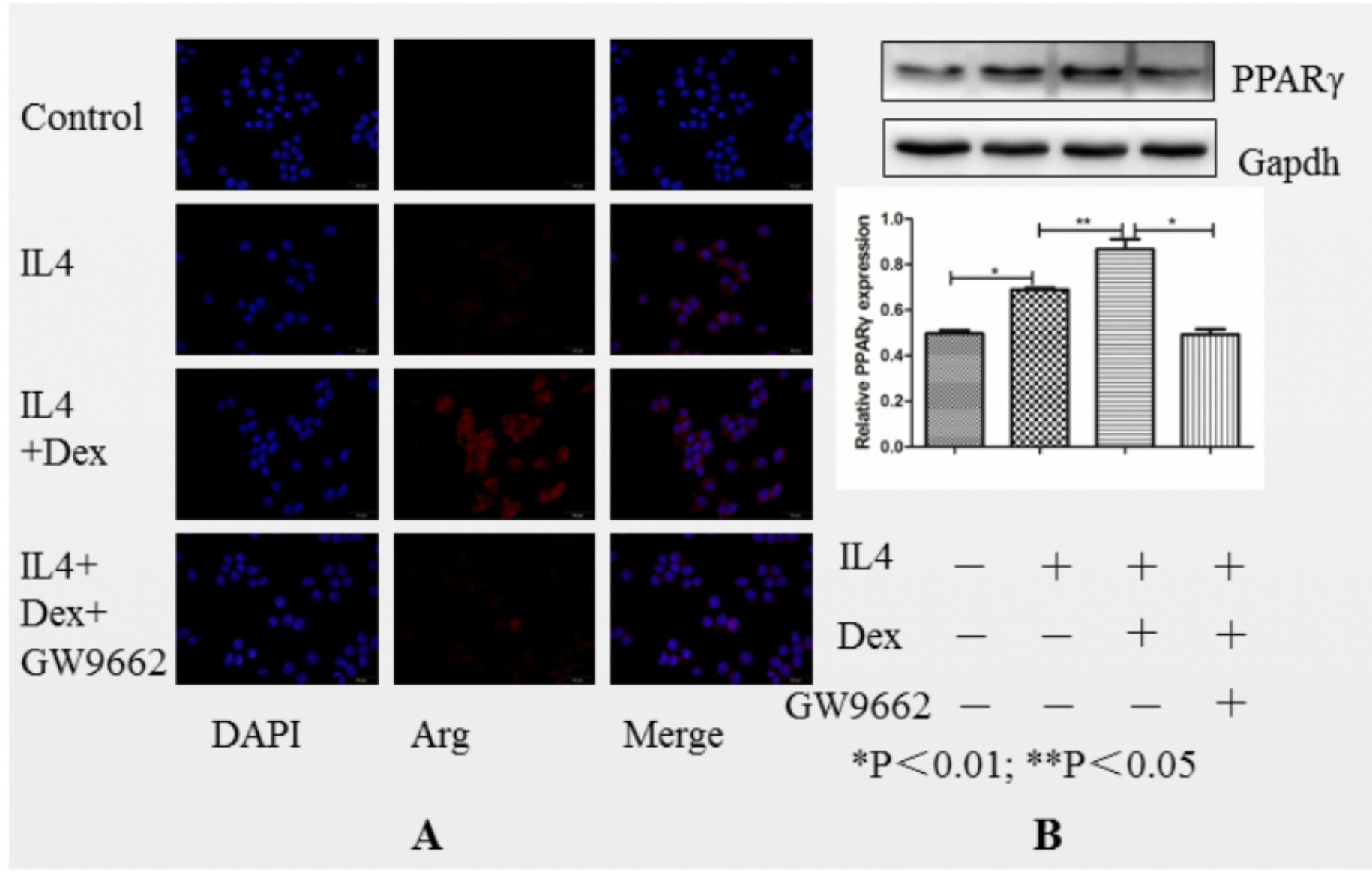

\section{Figure 3}

Dex enhances IL-4-induced PPARy activation. BV-2 cells were pretreated with Dex $(1 \mu \mathrm{M})$ or GW9662 (2 $\mu \mathrm{M})$. Nuclear extracts were prepared 24 hours after IL-4 treatment, and PPAR y protein levels were detected by Western blot. The representative results of three independent experiments are given. Grapdh was used to regulate the quantification of PPARy. The data showed the mean value of three independent experiments \pm SEM. The asterisk showed statistically significant difference ( $\left.{ }^{*}<0.01, * * P<0.05\right)$. 


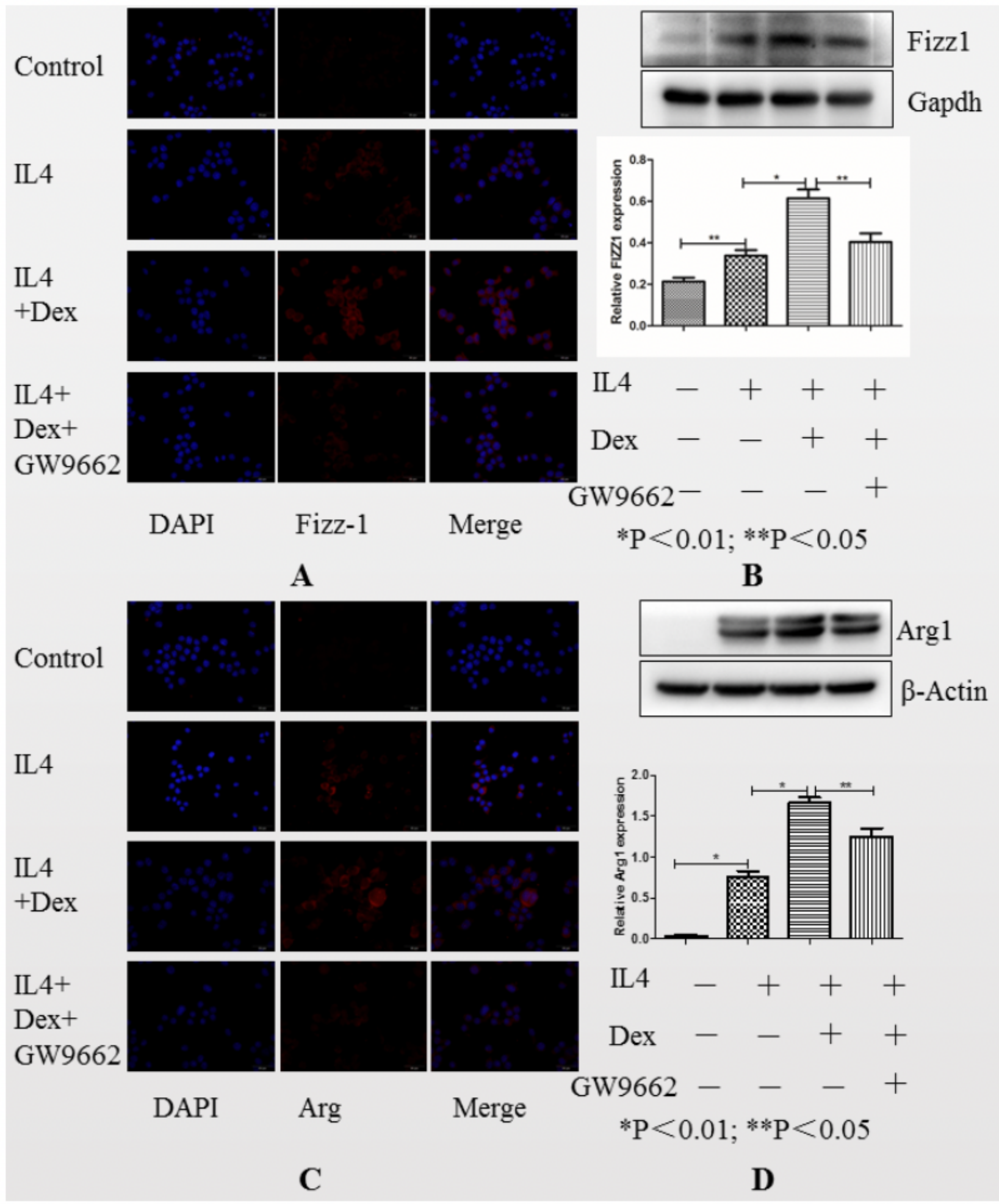

\section{Figure 4}

Inhibition of PPARy impacts microglia alternative activation induced by IL-4 and Dex. BV-2 cells were treated with $1 \mu \mathrm{M}$ Dex, $2 \mu \mathrm{M}$ GW9662, IL-4 $(20 \mathrm{ng} / \mathrm{ml})$ or combination of them. Levels of Arg1 and FIZZ1 were detected by Western blot 24 hours after IL-4 stimulation. A representative result from three independent experiments is shown. Quantification of Arg1 and FIZZ1 was normalized by $\beta$-actin and 
Grapdh respectively. Data are presented as mean \pm SEM for three independent experiments. Asterisks indicate significant difference $(* P<0.01$, ** $P \otimes 0.05)$.

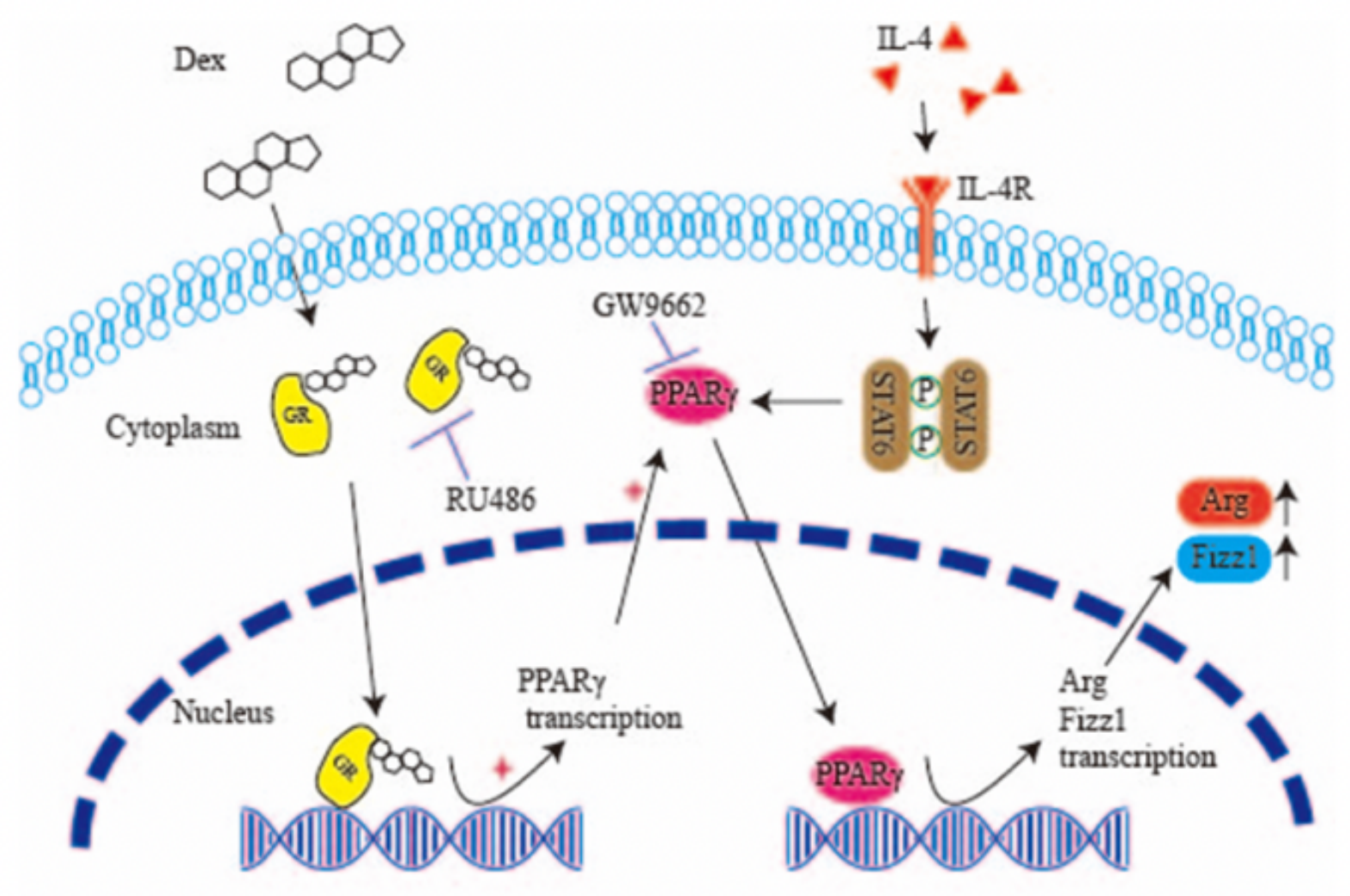

Figure 5

Schematic diagram of the effect of Dex on microglia replacement activation. Dex promotes IL-4-induced microglial selective activation and increases Arg1 and FIZZ1 expression through the STAT6/PPARY signaling pathway. 